Article

\title{
The Quality of Life of Patients with Hereditary Nonpolyposis Colorectal Cancer Undergoing Preoperative Chemoradiation
}

\author{
Valeriu Aurelian Chirica ${ }^{1}$, Mioara Matei ${ }^{1}$, Roxana Postolica ${ }^{2}$, Liliana Chelaru ${ }^{3}$, \\ Irina Mihaela Esanu ${ }^{4}$, Lidia Sanduleac ${ }^{5}$, Vlad Porumb 6,7, Mihaela Boanca 1,*, \\ Elena Adorata Coman ${ }^{1}$ and Doina Azoicai ${ }^{1}$
}

1 Department of Preventive Medicine and Interdisciplinarity, Faculty of Medicine, Grigore T. Popa University of Medicine and Pharmacy Iasi, 16 Universitatii Str., 700115 Iasi, Romania; valeriu-aurelian.chirica@umfiasi.ro (V.A.C.); mioara.matei@umfiasi.ro (M.M.); elena.coman@umfiasi.ro (E.A.C.); doina.azoicai@umfiasi.ro (D.A.)

2 Regional Institute of Oncology, 2-4 General Henri Mathias Berthelot Str., 700483 Iasi, Romania; roxana.a.cisman@d.umfiasi.ro

3 Department of Morphological Sciences, Faculty of Medicine, Grigore T. Popa University of Medicine and Pharmacy Iasi, 16 Universitatii Str., 700115 Iasi, Romania; liliana.chelaru@umfiasi.ro

4 Department of Internal Medicine, Faculty of Medicine, Grigore T. Popa University of Medicine and Pharmacy Iasi, 16 Universitatii Str., 700115 Iasi, Romania; irina.esanu@umfiasi.ro

5 Faculty of Medicine, 6th year student, Grigore T. Popa University of Medicine and Pharmacy Iasi, 16 Universitatii Str., 700115 Iasi, Romania; lidia.o.sanduleac@students.umfiasi.ro

6 Department of Surgery, Faculty of Medicine, Grigore T. Popa University of Medicine and Pharmacy Iasi, 16 Universitatii Str., 700115 Iasi, Romania; vlad.porumb@umfiasi.ro

7 Department of Surgery, Dr. Iacob Czihac Emergency Military Clinical Hospital Iasi, 7-9 General Henri Mathias Berthelot Str., 700483 Iasi, Romania

* Correspondence: boanca.mihaela@umfiasi.ro

Received: 8 August 2020; Accepted: 17 September 2020; Published: 21 September 2020

\begin{abstract}
Of all the neoplastic sites, colorectal cancer (CRC) is one of the most common cancers in the family. Studies estimate that approximately $30 \%$ of all the CRC cases are a hereditary form of the disease with a potentially high impact on the quality of life (QoL), with a high risk of recurrence, and with bio-psycho-social functioning. The aim of the study was to assess the QoL of colorectal cancer patients with genetic risk by using the European Organisation for Research and Treatment of Cancer (EORTC) questionnaire version QLQ-CR29. Materials and methods: The cross-sectional, single-center study was performed on a group of 32 patients with genetic risk of colorectal cancer, who underwent preoperative chemoradiation and surgery. Results: The series of values for the scores on the symptom scale varied between 15 and 30, and the average level of symptom scores did not differ significantly between gender ( 22.0 vs. $22.75 ; p=0.636)$, highlighting a moderate impairment of QoL. Scores for the emotional functioning scale were significantly lower in men (10.33 vs. 13.25; $p=0.049$ ), as were the scores for the physical functions ( 15.67 vs. $19.15 ; p=0.039)$, showing a decrease in QoL. Conclusions: The overall score showed an average QoL in patients with colorectal cancer with genetic risk, highlighting significant differences in psycho-emotional functioning between women and men.
\end{abstract}

Keywords: quality of life; chemoradiation; colorectal cancer; Lynch syndrome 


\section{Introduction}

Colorectal cancer (CRC) is one of the most commonly diagnosed cancers worldwide, with over 1.8 million new cases per year, of which approximately 500,000 new cases are in Europe [1]. CRC can be hereditary in $3 \%$ of patients, with an onset at younger ages [2,3]. The increasing incidence of CRC emphasized the studying of quality of life (QoL) in patients undergoing oncological and surgical therapies. The health deterioration due to cancer along with the consequences regarding psychological, physical or social functioning changes, all of them influencing the QoL [4]. Several studies highlighted the impact on QoL of CRC protocols in patients, immediately after chemoradiation and in the long run [5].

The QoL for cancer patients is associated with a greater number of factors [6], such as: socio-demographic characteristics; health-related factors; factors related to cancer and surgical procedures; lifestyle [7]; other factors [8,9]. The stage and location of CRC at the time of diagnosis is important in quantifying QoL, as they determine the symptoms, treatments and duration of therapy [10]. The stage I patients showed a positive progressive tendency in the QoL score. The stage IV patients had a negative score. In contrast, an initial decrease in QoL score, followed by better scores, was achieved by those with stage II and III, maybe because of a better perception of QoL after diagnosing CRC [11].

In patients with CRC undergoing surgery and colostomy, such procedures could lead to physical, psychological and social consequences on QoL. Studies showed an immediate decrease in QoL scores after surgical interventions, and a gradual recovery after 3 months. The presence of colostomy can lead to decreased QoL physical scores but also to emotional and social functioning scores as compared to those in patients with resection and anastomosis, but not all authors registered a significant difference [12]. Various studies worldwide investigated the role of chemotherapy and its associated symptoms influencing QoL in patients diagnosed and treated for CRC [13].

In Romania, the risk of developing cancer before the age of 75 is $23.1 \%$, of which $28.0 \%$ in men and $19.1 \%$ in women. The risk of dying from a form of cancer is $14.0 \%$, of which $19.1 \%$ in men and $9.8 \%$ in women. Colorectal cancer is the second most common cancer that affects the Romanian population (21,387,000 inhabitants), for both men and women. The number of new CRC cases reported in 2012 was 10,256 ( $13 \%$ of all cancers), of which 5760 were men ( $13.3 \%$ of all cancers in men) and 4496 were women (12.6\% of all cancers in women). In 2012 the number of deaths due to CRC was 5675 people, of which 3229 were men and 2446 were women. The 5 -year prevalence was 24,170 cases of CRC, of which 13,654 were men and 10,516 were women [10].

Of all the neoplastic sites, CRC is one of the most common familial cancers. People with Lynch syndrome are prone to various types of cancer, with a predilection for colonic and endometrial damage. Lynch syndrome accounts for $2-4 \%$ of all cases of CRC. Affected individuals may develop colonic adenomas with a higher frequency than the general population. The lifetime risk of developing a CRC is estimated at $50 \%$ [14].

Recent studies have shown that path_MLH1 and path_MSH2 carriers have a lifetime risk of CRC of approximately $50 \%$ and this incidence could not be decreased by surveillance colonoscopy. According to The Prospective Lynch Syndrome Database (PLSD), there was no risk of cancer in path_PMS2 carriers before the age of 40, and in older carriers, no other tumor associations were observed, excepting endometrial and prostate cancer [15]

In Lynch syndrome, malignant colonic polyps appear at an early age and have a more proximal location compared to those in sporadic CRC neoplasms. Histologically, these polyps are often poorly differentiated, mucinous and have a significant lymphocytic infiltrate. They are also characterized by a high level of microsatellite instability (MSI-H), a feature of tumors that occurs in the setting of genes involved in the DNA mismatch repair system (MMR system). Studies have shown that patients with colon cancer with MSI-H have a better prognosis compared to those without MSI [16]. 
Lynch syndrome is the result of a germline mutation in a class of genes involved in DNA mismatch repair (MMR), including $h M S H 2, h M L H 1, h M S H 6$ and $h P M S 2$. The MMR system is needed to maintain genomic stability by correcting single basic mismatches and insertion-deletion loops, which form during DNA replication [17].

According to the PLSD, a study conducted in Finland concluded that the increased incidence of CRC was linked to path_MLH1 carriers, but was not necessarily higher than other comparative studies have shown [18].

Another study carried out in Norway showed that the incidence of any localization in patients aged 40-70 years was 73\% in path_MLH1 and 76\% in path_MSH2 carriers, but lower (52\%) in path_MSH6 carriers [19].

The QoL questionnaire of the European Organization for Cancer Research and Treatment (EORTC) is an integrated type tool used to assess the QoL of cancer patients from a health point of view [14].

The aim of the study was to evaluate the QoL of patients with colorectal cancer with genetic risk who underwent preoperative cancer treatment (chemoradiation) and then underwent surgery, using an official questionnaire translated into Romanian.

\section{Materials and Methods}

The study group consisted of 32 patients who freely consented to participate in the research.

The cross-sectional study was conducted between November 2019 and March 2020 and interrupted temporarily due to the COVID-19 pandemic lockdown in Romania on March, 16th, 2020. The study was carried out at Regional Institute of Oncology, Iasi, Romania. The genetic risk was analyzed based on the genetic tree and the Amsterdam criteria by oncogenetics specialists. The inclusion criteria in the study were: patients over 18 years of age, without cognitive disorders, with unaltered judgment and introspection capacity, having awareness of oncological disease, with oncological diagnosis and genetic risk. All patients who were asked to answer the questionnaire, after basic information of the study, gave their informed consent and filled in the QoL assessment questionnaire. The exclusion criteria were: patients with oncological diagnosis but without a genetic risk, those in the stage of denial of the oncological disease, confused or disoriented, and those with problems in understanding the instructions to fill in the questionnaire.

The study was conducted in accordance with the Helsinki Declaration and with several published principles [20-26]. The inclusion criteria were suspected diagnosis of Lynch syndrome (Hereditary Nonpolyposis Colorectal Cancer), preoperative chemoradiation, and colostomy surgery. The exclusion criteria referred to the diagnosis of sporadic CRC, but they were not related to the age group, gender, or residence area of the patients.

Genetic risk was assessed taking into account the Amsterdam II Criteria (1998) for the identification of hereditary risk at CRC, also called criteria 3-2-1, as follows:

1. at least three persons in the same family affected by histologically confirmed cancer belonging to the HNPCC-Lynch spectrum (colorectal, endometrial, small bowel, urinary tract, renal pelvis, ovarian) histologically proven, united 2 by 2 by degree 1 of kinship (one of the persons is a 1st degree relative of the other two);

2. at least two generations are involved;

3. at least one of these cancers was declared before the age of 50 (familial adenomatous polyposis must be excluded).

The family history and the establishment of the genetic tree were traced (a key element for the evaluation of the notion of hereditary risk). 
For example, a person included in this study, from the point of view of genetic risk assessment, has the following family characteristics: 42-year-old patient diagnosed with rectal polyps; upper rectal adenocarcinoma-T1 N0 G1 M0; sigmoid diverticulosis at the age of 33 with a family history of colorectal cancer and polyps: mother-colonic polyp, brother-colonic diverticulosis, maternal aunt-colonic polyps, maternal grandfather-colon neoplasm, is accepted for genetic testing meeting the inclusion criteria. The features are provided in the Figure 1:

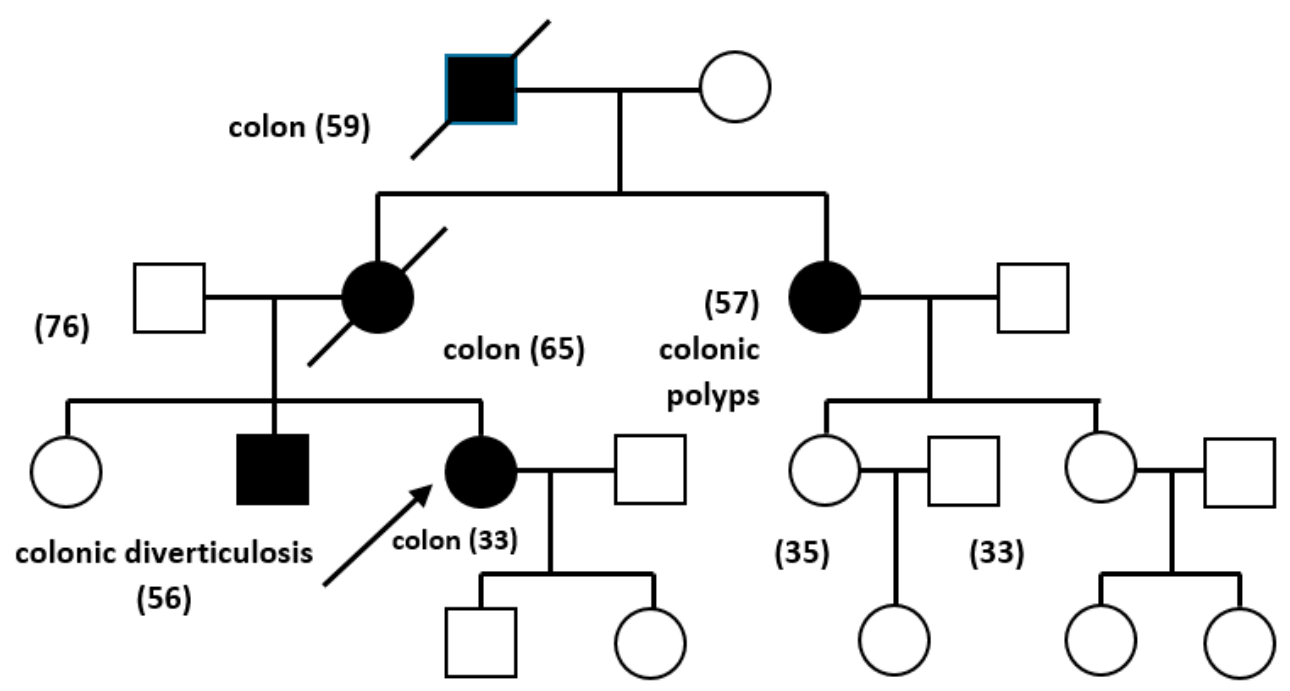

Figure 1. The genetic tree of a patient included in the present study.

The chemotherapy protocol consisted either of monotherapy with fluoropyrimidines (5-fluorouracil intravenously; capecitabine or tegafur-uracil, both administered orally) in combination with preoperative radiotherapy, or as combination therapy (5-fluorouracil, leucovorine, and oxaliplatin).

The cross-sectional study consisted of applying the EORTC questionnaire, version QLQ-CR29 specially designed for patients with CRC. The version translated in Romanian language was used online with the consent of the EORTC Organization, used for academic purposes only. The QLQ-CR-29 questionnaire has not been validated yet on the Romanian population, this being the purpose of a subsequent research based on the results of the present study. As of now there is only the official translation of the questionnaire in Romanian accepted by EORTC.

Questionnaire validation for the study group. Cronbach's alpha coefficient value was calculated separately by gender, so Cronbach's alpha coefficient in males was 0.819 and females was 0.907. Both values are above the threshold required to validate the use of the questionnaire (minimum value $=0.700$ ), thus the questionnaire was validated for the study group.

The principle of QLQ-CR29 scores consisted of applying to all cases the same questionnaire and the primary score was calculated for each case; the primary score was is standardized into two types of scores used by QLQ-CR29; the standardized scores ranged from 29 to 114, where a higher score represents a better functioning. The questionnaire subscales were the following: symptoms scale (scores ranged 15-30); functioning scale with the subsequent emotional scale (score ranged 5-20) and physical functioning including sex life scale (scores ranged 10-27). 


\section{Results}

The demographic data and tumor localization are presented in Table 1.

Table 1. Demographic data and tumor localization.

\begin{tabular}{cc}
\hline Variables & Statistics \\
\hline \multirow{2}{*}{ Age } & Range: $44-56$ \\
& Mean: 49.18 \\
& Most frequent group: $45-50(53.12 \%)$ \\
\hline \multirow{3}{*}{ Gender } & Male: $37.50 \%$ \\
& Memale: $62.50 \%$ \\
Residence area & Urban: $71.87 \%$ \\
& Rural: $28.13 \%$ \\
\hline \multirow{3}{*}{ Educational level } & U: R ratio $=2.55$ \\
& Compulsory: $15.62 \%$ \\
& Upper secondary: $37.50 \%$ \\
\hline \multirow{2}{*}{ Tumor localization } & Higher: $46.88 \%$ \\
& Sigmoid: $40.62 \%$ \\
& Rectosigmoid: $28.13 \%$ \\
& Rectal: $31.25 \%$ \\
\hline
\end{tabular}

\subsection{Symptoms Scale ("During the Past Week")}

Symptoms scale answers were added in Table 2.

Table 2. Symptoms scale.

\begin{tabular}{|c|c|c|c|c|c|c|c|c|}
\hline \multirow{2}{*}{$\begin{array}{c}\text { Items } \\
\text { I31. Urinary frequency/day }\end{array}$} & \multicolumn{2}{|c|}{ Not at All } & \multicolumn{2}{|c|}{ A Little } & \multicolumn{2}{|c|}{ Quite a Bit } & \multicolumn{2}{|c|}{ Very Much } \\
\hline & $\begin{array}{l}N=2 \\
6.30 \%\end{array}$ & $\begin{array}{c}M=50 \% \\
F=50 \%\end{array}$ & $\begin{array}{c}N=17 \\
53.1\end{array}$ & $\begin{array}{c}\mathrm{M}=70.6 \% \\
\mathrm{~F}=29,4 \%\end{array}$ & $\begin{array}{l}N=13 \\
40.60 \%\end{array}$ & $\begin{array}{c}M=53.8 \% \\
F=46.2 \%\end{array}$ & $\begin{array}{l}\mathrm{N}=0 \\
0.00 \%\end{array}$ & $\begin{array}{l}\mathrm{M}=0.0 \% \\
\mathrm{~F}=0.0 \%\end{array}$ \\
\hline I32. Urinary frequency/night & $\begin{array}{c}N=6 \\
18.80 \%\end{array}$ & $\begin{array}{c}M=83.3 \% \\
F=16.7 \%\end{array}$ & $\begin{array}{c}N=16 \\
50\end{array}$ & $\begin{array}{c}\mathrm{M}=81.3 \% \\
\mathrm{~F}=18.7 \%\end{array}$ & $\begin{array}{c}\mathrm{N}=9 \\
28.10 \%\end{array}$ & $\begin{array}{c}\mathrm{M}=24.2 \% \\
\mathrm{~F}=77.8 \%\end{array}$ & $\begin{array}{l}\mathrm{N}=1 \\
3.10 \%\end{array}$ & $\begin{array}{c}M=0.0 \% \\
F=100.0 \%\end{array}$ \\
\hline I33. Urinary incontinence & $\begin{array}{l}N=23 \\
71.90 \%\end{array}$ & $\begin{array}{c}\mathrm{M}=65.2 \% \\
\mathrm{~F}=34.8 \%\end{array}$ & $\begin{array}{c}\mathrm{N}=9 \\
28.10 \%\end{array}$ & $\begin{aligned} \mathrm{M} & =55.6 \% \\
\mathrm{~F} & =4.4 \%\end{aligned}$ & $\begin{array}{l}\mathrm{N}=0 \\
0.00 \%\end{array}$ & $\begin{array}{c}\mathrm{M}=0.0 \% \\
\mathrm{~F}=0.0 \%\end{array}$ & $\begin{array}{l}\mathrm{N}=0 \\
0.00 \%\end{array}$ & $\begin{array}{c}\mathrm{M}=0.0 \% \\
\mathrm{~F}=0.0 \%\end{array}$ \\
\hline I34. Dysuria & $\begin{array}{l}N=20 \\
59.40 \%\end{array}$ & $\begin{aligned} \mathrm{M} & =52.6 \% \\
\mathrm{~F} & =7.4 \%\end{aligned}$ & $\begin{array}{c}\mathrm{N}=9 \\
28.10 \%\end{array}$ & $\begin{array}{c}\mathrm{M}=77.8 \% \\
\mathrm{~F}=22.2 \%\end{array}$ & $\begin{array}{c}\mathrm{N}=4 \\
12.50 \%\end{array}$ & $\begin{array}{c}M=75.0 \% \\
F=25.0 \%\end{array}$ & $\begin{array}{l}\mathrm{N}=0 \\
0.00 \%\end{array}$ & $\begin{array}{l}\mathrm{M}=0.0 \% \\
\mathrm{~F}=0.0 \%\end{array}$ \\
\hline I35. Abdominal pain & $\begin{array}{l}N=13 \\
40.60 \%\end{array}$ & $\begin{array}{c}M=53.8 \% \\
F=46.2 \%\end{array}$ & $\begin{array}{c}\mathrm{N}=6 \\
37.50 \%\end{array}$ & $\begin{array}{c}M=73.3 \% \\
F=26.7 \%\end{array}$ & $\begin{array}{l}N=12 \\
18.80 \%\end{array}$ & $\begin{array}{c}M=83.3 \% \\
F=16.7 \%\end{array}$ & $\begin{array}{l}\mathrm{N}=1 \\
3.10 \%\end{array}$ & $\begin{array}{c}\mathrm{M}=0.0 \% \\
\mathrm{~F}=100.0 \%\end{array}$ \\
\hline I36. Buttock pain & $\begin{array}{l}\mathrm{N}=12 \\
37.50 \%\end{array}$ & $\begin{array}{c}\mathrm{M}=41.7 \% \\
\mathrm{~F}=58.3 \%\end{array}$ & $\begin{array}{l}N=15 \\
46.90 \%\end{array}$ & $\begin{array}{c}M=73.3 \% \\
F=26.7 \%\end{array}$ & $\begin{array}{c}N=5 \\
15.60 \%\end{array}$ & $\begin{array}{c}M=80.0 \% \\
F=20.0 \%\end{array}$ & $\begin{array}{l}N=0 \\
0.00 \%\end{array}$ & $\begin{array}{c}\mathrm{M}=0.0 \% \\
\mathrm{~F}=0.0 \%\end{array}$ \\
\hline I37. Bloated feeling & $\begin{array}{c}\mathrm{N}=6 \\
18.10 \%\end{array}$ & $\begin{array}{c}\mathrm{M}=33.3 \% \\
\mathrm{~F}=66.7 \%\end{array}$ & $\begin{array}{c}N=19 \\
78.15\end{array}$ & $\begin{array}{c}M=63.2 \% \\
F=36.8 \%\end{array}$ & $\begin{array}{c}\mathrm{N}=7 \\
21.90 \%\end{array}$ & $\begin{array}{c}M=85.7 \% \\
F=14.3 \%\end{array}$ & $\begin{array}{l}\mathrm{N}=0 \\
0.00 \%\end{array}$ & $\begin{array}{c}\mathrm{M}=0.0 \% \\
\mathrm{~F}=0.0 \%\end{array}$ \\
\hline I38. Blood in stools & $\begin{array}{l}N=16 \\
50.00 \%\end{array}$ & $\begin{array}{c}M=50 \% \\
F=50 \%\end{array}$ & $\begin{array}{l}N=14 \\
43.70 \%\end{array}$ & $\begin{array}{c}\mathrm{M}=78.6 \% \\
\mathrm{~F}=21.4 \%\end{array}$ & $\begin{array}{l}\mathrm{N}=2 \\
6.30 \%\end{array}$ & $\begin{array}{c}M=50 \% \\
F=50 \%\end{array}$ & $\begin{array}{l}\mathrm{N}=0 \\
0.00 \%\end{array}$ & $\begin{array}{l}\mathrm{M}=0.0 \% \\
\mathrm{~F}=0.0 \%\end{array}$ \\
\hline I39. Mucus in stools & $\begin{array}{l}N=16 \\
50.00 \%\end{array}$ & $\begin{array}{c}\mathrm{M}=56.3 \% \\
\mathrm{~F}=43.8 \%\end{array}$ & $\begin{array}{l}\mathrm{N}=16 \\
50.00 \%\end{array}$ & $\begin{array}{c}\mathrm{M}=58.8 \% \\
\mathrm{~F}=41.2 \%\end{array}$ & $\begin{array}{l}\mathrm{N}=0 \\
0.00 \%\end{array}$ & $\begin{array}{c}\mathrm{M}=0.0 \% \\
\mathrm{~F}=0.0 \%\end{array}$ & $\begin{array}{l}\mathrm{N}=0 \\
0.00 \%\end{array}$ & $\begin{array}{c}\mathrm{M}=0.0 \% \\
\mathrm{~F}=0.0 \%\end{array}$ \\
\hline I40. Dry mouth & $\begin{array}{c}\mathrm{N}=4 \\
12.50 \%\end{array}$ & $\begin{array}{c}M=0.0 \% \\
F=100.0 \%\end{array}$ & $\begin{array}{l}N=20 \\
62.50 \%\end{array}$ & $\begin{array}{c}\mathrm{M}=60.0 \% \\
\mathrm{~F}=40.0 \%\end{array}$ & $\begin{array}{c}N=6 \\
18.80 \%\end{array}$ & $\begin{array}{c}M=50 \% \\
F=50 \%\end{array}$ & $\begin{array}{l}\mathrm{N}=2 \\
6.30 \%\end{array}$ & $\begin{array}{c}M=50 \% \\
F=50 \%\end{array}$ \\
\hline I41. Hair loss & $\begin{array}{c}\mathrm{N}=8 \\
25.00 \%\end{array}$ & $\begin{array}{c}\mathrm{M}=37.5 \% \\
\mathrm{~F}=62.5 \%\end{array}$ & $\begin{array}{l}N=13 \\
40.60 \%\end{array}$ & $\begin{array}{c}\mathrm{M}=76.9 \% \\
\mathrm{~F}=23.1 \%\end{array}$ & $\begin{array}{c}\mathrm{N}=7 \\
21.90 \%\end{array}$ & $\begin{array}{l}M=71 \% \\
F=28.6 \%\end{array}$ & $\begin{array}{l}N=4 \\
12.50 \%\end{array}$ & $\begin{array}{c}M=50 \% \\
F=50 \%\end{array}$ \\
\hline I42. Trouble with taste & $\begin{array}{l}N=10 \\
31.30 \%\end{array}$ & $\begin{array}{c}M=50 \% \\
F=50 \%\end{array}$ & $\begin{array}{l}\mathrm{N}=12 \\
37.50 \%\end{array}$ & $\begin{array}{c}\mathrm{M}=75.0 \% \\
\mathrm{~F}=25.0 \%\end{array}$ & $\begin{array}{c}\mathrm{N}=9 \\
28.10 \%\end{array}$ & $\begin{array}{c}\mathrm{M}=55.6 \% \\
\mathrm{~F}=44.4 \%\end{array}$ & $\begin{array}{l}\mathrm{N}=1 \\
3.10 \%\end{array}$ & $\begin{array}{c}\mathrm{M}=100.0 \% \\
\mathrm{~F}=0.0 \%\end{array}$ \\
\hline
\end{tabular}


By applying the Kruskal-Wallis significance tests, the correlation matrix of the items for symptoms scale highlighted the following aspects of significance for the purpose of our study: urination at night correlated significantly with abdominal pain $(p=0.015)$ and loss of taste $(p=0.011)$; urination pain was significantly correlated with abdominal pain $(p=0.002)$, blood stool $(p=0.007)$, and loss of taste $(p=0.017)$; the bloating sensation was significantly correlated with hair loss $(p=0.021)$ and taste loss $(p=0.05)$; the presence of blood in stools was significantly correlated with hair loss $(p=0.047)$ and taste loss $(p=0.03)$; hair loss was significantly correlated with taste loss $(p=0.037)$.

Symptoms score. The symptoms scores values ranged from 15 to 30, with a mean of 22.47; the mean level did not differ significantly between genders (22.0 vs. $22.75 ; p=0.636)$, showing a moderate impairment of the QoL (Table 3, Figure 2).

Table 3. Descriptive statistics of symptoms score by gender.

\begin{tabular}{|c|c|c|c|c|c|c|c|c|c|}
\hline \multirow[t]{2}{*}{ Gender } & \multirow[t]{2}{*}{$\mathbf{N}$} & \multirow[t]{2}{*}{ Mean } & \multirow{2}{*}{$\begin{array}{c}\text { Std. } \\
\text { Deviation }\end{array}$} & \multirow{2}{*}{$\begin{array}{l}\text { Std. } \\
\text { Error }\end{array}$} & \multicolumn{2}{|c|}{$\begin{array}{l}\text { 95\%Confidence } \\
\text { Interval }\end{array}$} & \multirow[t]{2}{*}{ Min } & \multirow[t]{2}{*}{ Max } & \multirow{2}{*}{$\begin{array}{c}\text { F Test } \\
\text { (ANOVA) } \\
p\end{array}$} \\
\hline & & & & & $-95 \% \mathrm{CI}$ & $+95 \% \mathrm{CI}$ & & & \\
\hline Male & 12 & 22.00 & 4.02 & 1.16 & 19.44 & 24.56 & 17 & 28 & \multirow{3}{*}{0.636} \\
\hline Female & 20 & 22.75 & 4.45 & 0.99 & 20.67 & 24.83 & 15 & 30 & \\
\hline Total & 32 & 22.47 & 4.24 & 0.75 & 20.94 & 24.00 & 15 & 30 & \\
\hline
\end{tabular}

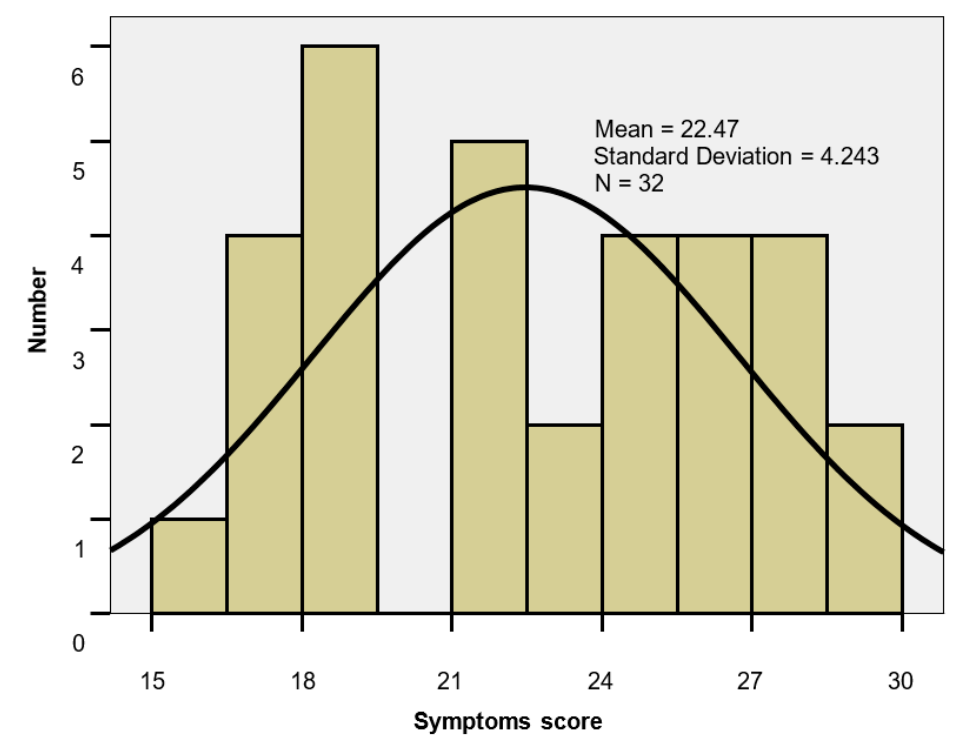

Figure 2. Symptoms score histogram.

\subsection{Functioning Scale ("during the Past Week")}

\subsubsection{Emotional Functioning Scale}

Emotional functioning scale answers were added in Table 4 . 
Table 4. Emotional functioning scale.

\begin{tabular}{|c|c|c|c|c|c|c|c|c|}
\hline \multirow{2}{*}{$\frac{\text { Items }}{\text { I43. Anxiety }}$} & \multicolumn{2}{|c|}{ Not at All } & \multicolumn{2}{|c|}{ A Little } & \multicolumn{2}{|c|}{ Quite a Bit } & \multicolumn{2}{|c|}{ Very Much } \\
\hline & $\begin{array}{l}N=1 \\
3.10 \%\end{array}$ & $\begin{array}{c}M=0.0 \% \\
F=100.0 \%\end{array}$ & $\begin{array}{l}\mathrm{N}=10 \\
31.30 \%\end{array}$ & $\begin{array}{l}\mathrm{M}=40.0 \% \\
\mathrm{~F}=60.0 \%\end{array}$ & $\begin{array}{l}\mathrm{N}=12 \\
37.50 \%\end{array}$ & $\begin{array}{c}\mathrm{M}=75.0 \% \\
\mathrm{~F}=25.0 \%\end{array}$ & $\begin{array}{c}\mathrm{N}=9 \\
28.10 \%\end{array}$ & $\begin{array}{l}\mathrm{M}=77.9 \% \\
\mathrm{~F}=22.2 \%\end{array}$ \\
\hline I44. Weight & $\begin{array}{c}\mathrm{N}=7 \\
21.90 \%\end{array}$ & $\begin{array}{l}M=57.1 \% \\
F=42.9 \%\end{array}$ & $\begin{array}{l}N=12 \\
37.50 \%\end{array}$ & $\begin{array}{c}M=50 \% \\
F=50 \%\end{array}$ & $\begin{array}{l}\mathrm{N}=10 \\
31.30 \%\end{array}$ & $\begin{array}{l}M=70.0 \% \\
F=30.0 \%\end{array}$ & $\begin{array}{l}\mathrm{N}=3 \\
9.40 \%\end{array}$ & $\begin{array}{c}M=100.0 \% \\
F=0.0 \%\end{array}$ \\
\hline $\begin{array}{c}\text { I45. Body } \\
\text { image/attractiveness }\end{array}$ & $\begin{array}{c}\mathrm{N}=8 \\
25.00 \%\end{array}$ & $\begin{array}{l}M=25.0 \% \\
F=75.0 \%\end{array}$ & $\begin{array}{l}\mathrm{N}=9 \\
28.10 \%\end{array}$ & $\begin{array}{l}M=77.8 \% \\
F=22.2 \%\end{array}$ & $\begin{array}{l}\mathrm{N}=11 \\
34.40 \%\end{array}$ & $\begin{array}{l}M=72.7 \% \\
F=27.3 \%\end{array}$ & $\begin{array}{c}\mathrm{N}=4 \\
12.50 \%\end{array}$ & $\begin{array}{l}\mathrm{M}=75.0 \% \\
\mathrm{~F}=25.0 \%\end{array}$ \\
\hline I46. Body image/gender & $\begin{array}{l}\mathrm{N}=12 \\
37.50 \%\end{array}$ & $\begin{array}{c}M=33.3 \% \\
F=66.7 \%\end{array}$ & $\begin{array}{c}\mathrm{N}=7 \\
21.90 \%\end{array}$ & $\begin{array}{c}M=85.7 \% \\
F=14.3 \%\end{array}$ & $\begin{array}{c}N=8 \\
25.00 \%\end{array}$ & $\begin{array}{l}M=87.5 \% \\
F=12.5 \%\end{array}$ & $\begin{array}{l}N=5 \\
15.60 \%\end{array}$ & $\begin{array}{l}\mathrm{M}=60.0 \% \\
\mathrm{~F}=40.0 \%\end{array}$ \\
\hline $\begin{array}{c}\text { I47. Body } \\
\text { image/satisfaction }\end{array}$ & $\begin{array}{l}N=10 \\
31.30 \%\end{array}$ & $\begin{array}{c}M=50 \% \\
F=50 \%\end{array}$ & $\begin{array}{l}\mathrm{N}=6 \\
18.70 \%\end{array}$ & $\begin{array}{l}M=66.7 \% \\
F=33.3 \%\end{array}$ & $\begin{array}{c}N=8 \\
25.00 \%\end{array}$ & $\begin{array}{l}\mathrm{M}=62.5 \% \\
\mathrm{~F}=37.5 \%\end{array}$ & $\begin{array}{c}\mathrm{N}=8 \\
25.00 \%\end{array}$ & $\begin{array}{l}\mathrm{M}=75.0 \% \\
\mathrm{~F}=25.0 \%\end{array}$ \\
\hline
\end{tabular}

By applying Kruskal-Wallis significance tests, the correlation matrix of items for emotional functioning scale revealed strong and statistically significant correlations between the patient's worries for future health, weight, attractiveness, and masculinity/femininity, generally related to one's body $(p<0.05)$.

\subsubsection{Emotional Functioning Score}

The scores values for emotional functioning scale ranged between five and 20. The scores values were homogeneous, with the mean level lower in men than in women (10.33 vs. 13.25; $p=0.049)$, highlighting a decrease in QoL of patients suffering from CRC (Table 5, Figure 3).

Table 5. Descriptive statistics of emotional functioning score by gender.

\begin{tabular}{|c|c|c|c|c|c|c|c|c|c|}
\hline \multirow[t]{2}{*}{ Gender } & \multirow[t]{2}{*}{$\mathbf{N}$} & \multirow[t]{2}{*}{ Mean } & \multirow{2}{*}{$\begin{array}{c}\text { Std. } \\
\text { Deviation }\end{array}$} & \multirow{2}{*}{$\begin{array}{l}\text { Std. } \\
\text { Error }\end{array}$} & \multicolumn{2}{|c|}{$\begin{array}{l}\text { 95\% Confidence } \\
\text { Interval }\end{array}$} & \multirow[t]{2}{*}{ Min } & \multirow[t]{2}{*}{$\operatorname{Max}$} & \multirow{2}{*}{$\begin{array}{c}\text { F Test } \\
\text { (ANOVA) } \\
p\end{array}$} \\
\hline & & & & & $-95 \% \mathrm{CI}$ & $+95 \% \mathrm{CI}$ & & & \\
\hline Male & 12 & 10.33 & 4.58 & 1.32 & 7.42 & 13.24 & 5 & 19 & \multirow{3}{*}{0.049} \\
\hline Female & 20 & 13.25 & 4.01 & 0.89 & 11.37 & 15.13 & 6 & 20 & \\
\hline Total & 32 & 12.16 & 4.40 & 0.78 & 10.57 & 13.74 & 5 & 20 & \\
\hline
\end{tabular}

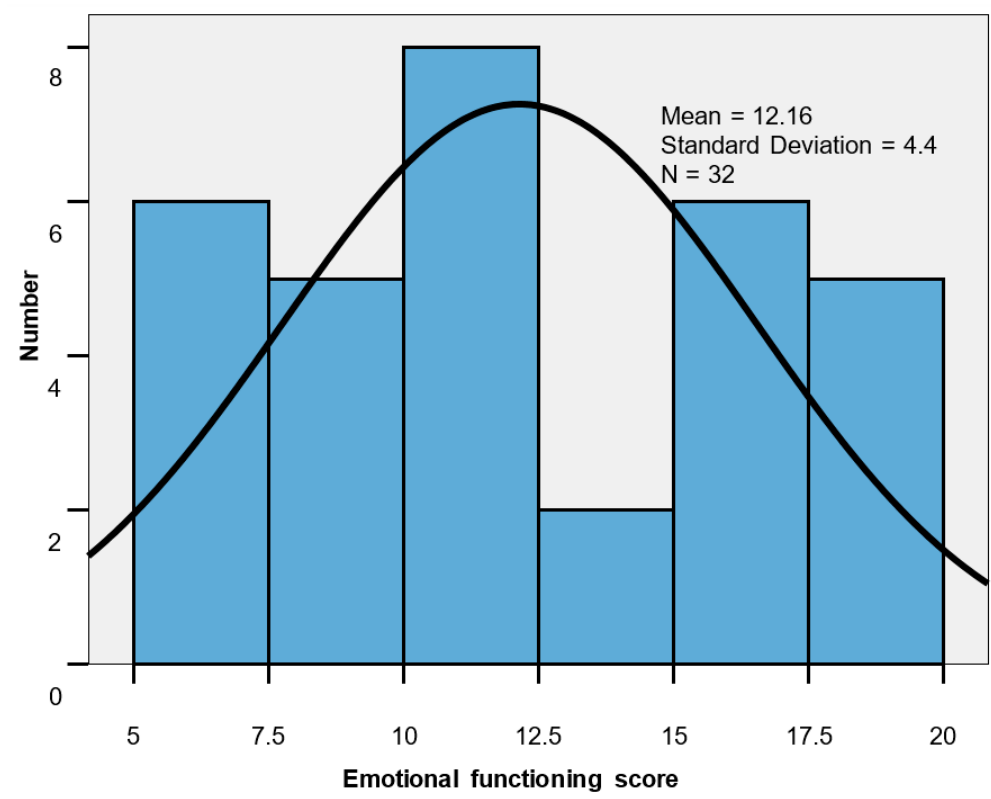

Figure 3. Emotional functioning score histogram 
3.2.3. Physical Functioning Scale

The physical functioning scale answers are shown in Table 6 .

Table 6. Physical functioning scale.

\begin{tabular}{ccccc}
\hline Items & Not at All & A Little & Quite a Bit & Very Much \\
\hline \multirow{2}{*}{ I49. Stoma/unintentional defecation } & $\mathrm{N}=0$ & $\mathrm{~N}=19$ & $\mathrm{~N}=13$ & $\mathrm{~N}=0$ \\
& $0.00 \%$ & $60.00 \%$ & 40 & $0.00 \%$ \\
\hline \multirow{2}{*}{ I50. Stoma/stools leakage } & $\mathrm{N}=2$ & $\mathrm{~N}=20$ & $\mathrm{~N}=6$ & $\mathrm{~N}=3$ \\
& $9.30 \%$ & $70.00 \%$ & $20.00 \%$ & $10.00 \%$ \\
\hline \multirow{2}{*}{ I51. Stoma/sore skin } & $\mathrm{N}=0$ & $\mathrm{~N}=13$ & $\mathrm{~N}=19$ & $\mathrm{~N}=0$ \\
& $0.00 \%$ & 40.05 & $60.00 \%$ & $0.00 \%$ \\
\hline \multirow{2}{*}{ I52. Stoma/bag changes/day } & $\mathrm{N}=6$ & $\mathrm{~N}=19$ & $\mathrm{~N}=7$ & $\mathrm{~N}=0$ \\
& $20.00 \%$ & $60.00 \%$ & $20.00 \%$ & $0.00 \%$ \\
\hline \multirow{2}{*}{ I53. Stoma/bag changes/night } & $\mathrm{N}=0$ & $\mathrm{~N}=16$ & $\mathrm{~N}=16$ & $\mathrm{~N}=0$ \\
& $0.00 \%$ & $50.00 \%$ & $50.00 \%$ & $0.00 \%$ \\
\hline \multirow{2}{*}{ I54. Stoma/embarrassment } & $\mathrm{N}=0$ & $\mathrm{~N}=6$ & $\mathrm{~N}=16$ & $\mathrm{~N}=10$ \\
& $0.00 \%$ & $20.00 \%$ & $50.00 \%$ & $30.00 \%$ \\
\hline \multirow{2}{*}{ I55. Stoma care/problems } & $\mathrm{N}=3$ & $\mathrm{~N}=10$ & $\mathrm{~N}=13$ & $\mathrm{~N}=6$ \\
& $10.00 \%$ & $30.00 \%$ & $40.00 \%$ & $20.00 \%$ \\
\hline Sex life scale & & & & \\
\hline \multirow{2}{*}{ I56 (male). Sex interest } & $\mathrm{N}=10$ & $\mathrm{~N}=22$ & $\mathrm{~N}=0$ & $\mathrm{~N}=0$ \\
& $33.30 \%$ & $66.70 \%$ & $0.00 \%$ & $0.00 \%$ \\
\hline \multirow{2}{*}{ I57 (male). Erection problems } & $\mathrm{N}=16$ & $\mathrm{~N}=8$ & $\mathrm{~N}=3$ & $\mathrm{~N}=5$ \\
& $50.00 \%$ & $25.00 \%$ & $8.30 \%$ & $16.70 \%$ \\
\hline \multirow{2}{*}{ (female). Intercourse discomfort } & $\mathrm{N}=1$ & $\mathrm{~N}=13$ & $\mathrm{~N}=2$ & $\mathrm{~N}=3$ \\
& $25.00 \%$ & $15.00 \%$ & $10.00 \%$ & $50.00 \%$ \\
\hline
\end{tabular}

By applying the Kruskal-Wallis significance tests, the correlation matrix of items for physical functioning scale in the QLQ-CR29 questionnaire highlighted the following significant aspects for our study: stool leakage was significantly correlated with a sore skin $(p=0.001)$, an accelerated intestinal transit during day $(p=0.024)$ or night $(p=0.047)$ and with the embarrassment to wear a stoma bag $(p=0.018)$, and with a decreased interest for sex $(p=0.006)$.

Additional data regarding statistical analysis results are provided as Supplementary Materials.

\subsubsection{Physical Functioning Score}

The scores values for the physical functioning scale ranged 10-27; the group mean $17.84 \pm 4.66$ was close to the median value of 18 . The scores mean level was significantly lower in men (15.67 vs. $19.15 ; p=0.039$ ), indicating an important impairment of physical functioning (Table 7 , Figure 4). 
Table 7. Descriptive statistics of physical functioning score by gender.

\begin{tabular}{|c|c|c|c|c|c|c|c|c|c|}
\hline \multirow[t]{2}{*}{ Gender } & \multirow[t]{2}{*}{$\mathbf{N}$} & \multirow[t]{2}{*}{ Mean } & \multirow{2}{*}{$\begin{array}{c}\text { Std. } \\
\text { Deviation }\end{array}$} & \multirow{2}{*}{$\begin{array}{l}\text { Std. } \\
\text { Error }\end{array}$} & \multicolumn{2}{|c|}{$\begin{array}{l}\text { 95\% Confidence } \\
\text { Interval }\end{array}$} & \multirow[t]{2}{*}{ Min } & \multirow[t]{2}{*}{$\operatorname{Max}$} & \multirow{2}{*}{$\begin{array}{c}\text { F Test } \\
\text { (ANOVA) } \\
p\end{array}$} \\
\hline & & & & & $-95 \% \mathrm{CI}$ & $+95 \% \mathrm{CI}$ & & & \\
\hline Male & 12 & 15.67 & 3.17 & 0.92 & 13.65 & 17.68 & 11 & 20 & \multirow{3}{*}{0.039} \\
\hline Female & 20 & 19.15 & 4.99 & 1.12 & 16.82 & 21.48 & 10 & 27 & \\
\hline Total & 32 & 17.84 & 4.66 & 0.82 & 16.16 & 19.53 & 10 & 27 & \\
\hline
\end{tabular}

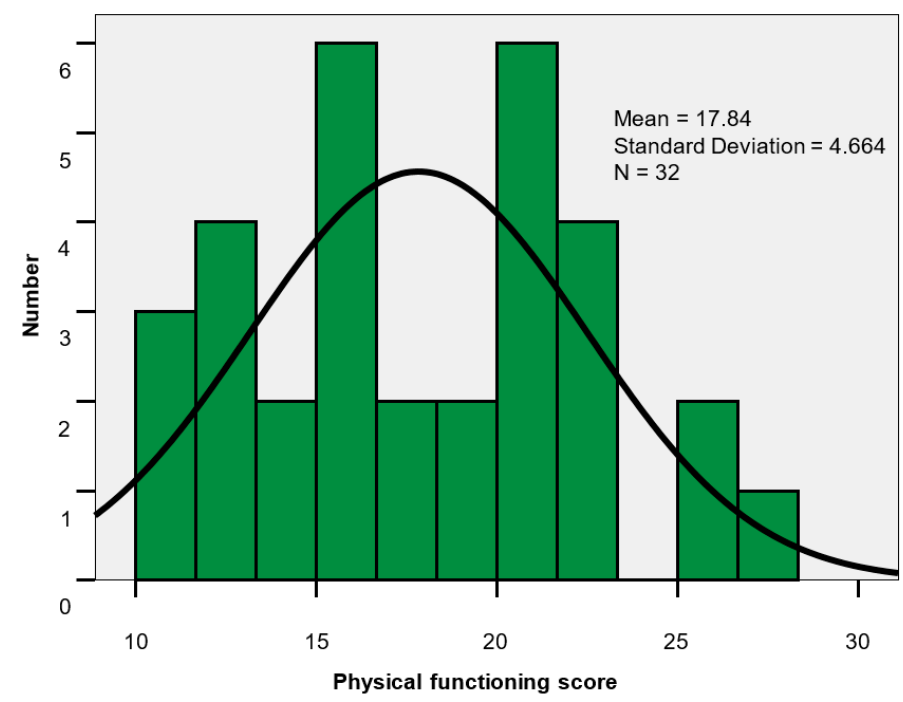

Figure 4. Physical functioning score histogram.

\subsubsection{Overall Score for QoL}

The set of values for the overall score ranged between 33 and 76; group mean $52.47 \pm 11.40$. The score mean level was lower in men than in women ( 48.0 vs. $55.15 ; p=0.086)$, indicating a more important impairment of QoL in male patients (Table 8, Figure 5).

Table 8. Descriptive statistics of the overall score by gender.

\begin{tabular}{|c|c|c|c|c|c|c|c|c|c|}
\hline \multirow[t]{2}{*}{ Gender } & \multirow[t]{2}{*}{$\mathbf{N}$} & \multirow[t]{2}{*}{ Mean } & \multirow{2}{*}{$\begin{array}{c}\text { Std. } \\
\text { Deviation }\end{array}$} & \multirow{2}{*}{$\begin{array}{l}\text { Std. } \\
\text { Error }\end{array}$} & \multicolumn{2}{|c|}{$\begin{array}{l}\text { 95\% Confidence } \\
\text { Interval }\end{array}$} & \multirow[t]{2}{*}{ Min } & \multirow[t]{2}{*}{ Max } & \multirow{2}{*}{$\begin{array}{c}\text { F Test } \\
\text { (ANOVA) } \\
p\end{array}$} \\
\hline & & & & & $-95 \%$ CI & $+95 \% \mathrm{CI}$ & & & \\
\hline Male & 12 & 48.00 & 8.88 & 2.56 & 42.36 & 53.64 & 37 & 63 & \multirow{3}{*}{0.086} \\
\hline Female & 20 & 55.15 & 12.09 & 2.70 & 49.49 & 60.81 & 33 & 76 & \\
\hline Total & 32 & 52.47 & 11.40 & 2.02 & 48.36 & 56.58 & 33 & 76 & \\
\hline
\end{tabular}




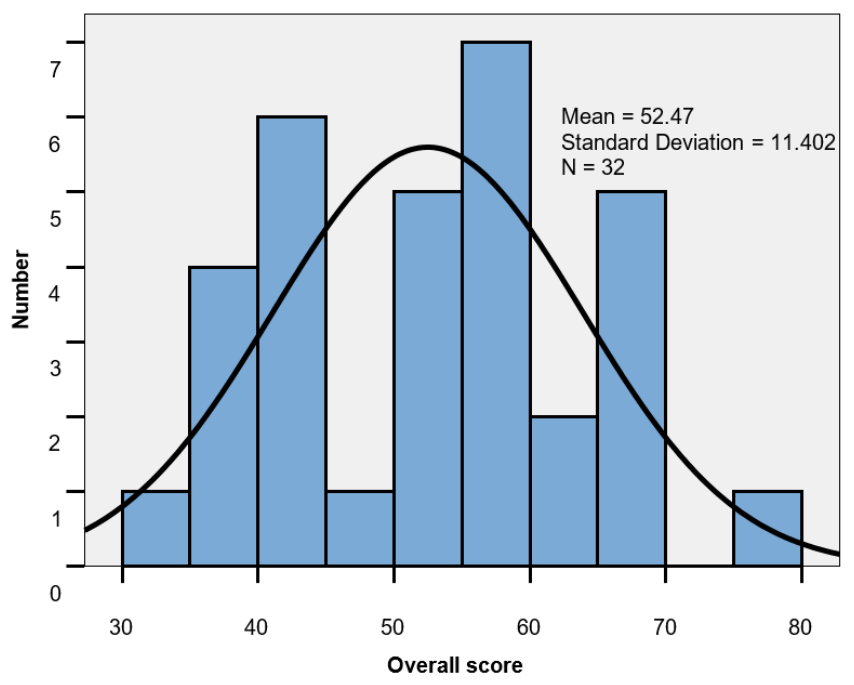

Figure 5. Global score histogram.

\section{Discussion}

The modern approach of treatment options for patients with CRC leads to the necessity of taking into consideration their QoL after surgical and/or oncological protocol application. QoL should be included in the evaluation of such patients, along with the survival assessment, tumor recurrence, side effects and toxicity of chemoradiation, the physical and psychological effects of wearing a stoma, as well as the cost-effectiveness of procedures.

We consider that the main statements of our study are the following: the average level of scores for symptoms did not differ significantly between the sexes, highlighting a moderate impairment of QoL. Men did not feel less masculine, while women felt less feminine as a result of illness and treatment; the correlation matrix of items for emotional function showed strong correlations, statistically significant between the patient's concerns for future health, weight, feelings of dissatisfaction with attractiveness, femininity/masculinity and, generally, with their own body; the symptoms characteristic of discomfort caused by the colostomy bag or unintentional manifestations generated by bowel movement showed correlations that led the patient to reanalyze his/her physical and emotional effects, and greater impairment of physical and emotional functions was noted in men.

Scores are transformed linearly, giving a score between zero and 100. The results of our study were compared to the results of other studies that showed that the higher the scores, the better they function on the functioning scales and a higher level of symptoms on the symptoms scale [27].

The results of the present study will be used in the calibration of the Romanian population and the construction of their own standards for patients with CRC with a genetic risk.

The physical and emotional changes in patients with stoma varied by gender. In female patients, a lower QoL score was reported for emotional and physical functioning, but in males, we described, just like other studies [28], a decrease in the score. These findings can lead to a gradual reduction in a person's confidence and can change his/her social functioning as well [5]. The impact of colostomy, together with chemoradiation and other therapeutic procedures in patients diagnosed with CRC, could be ameliorated by providing specialized assistance, such as colostomy care programs and psychological counseling [29].

In a study on patients with CRC and their partners, regarding the influence of oncological treatments on psychological functioning, the authors showed significant differences in anxiety, depression and traumatic stress symptoms among patients who underwent surgery, and chemoradiation, as compared to patients treated only surgically. The first group experienced higher anxiety scores, more depressive symptoms and more posttraumatic stress symptoms [30]. 
In a prospective longitudinal study, authors demonstrated that the group activities of patients with CRC and colostomy, sharing personal experiences, would decrease the level of isolation and feeling of loneliness caused by the stoma wearing, along with the diagnosis of cancer, and chemoradiation [31].

The increased toxicity of chemotherapeutic substances contributes to smaller or more severe side effects, influencing the QoL of oncological patients. However, these side effects are not only given by the pharmacodynamics of the chemicals, but they can also be influenced by individual perception and other psychological factors related to the patients [32].

The limitations of the QoL studies in patients with CRC could be the lack of accumulation of information by systematic reviews and the lack of a gold standard for QoL measurement. Other limitations of such studies could be due to poor data acquisition, low response rates, a reduced sample size, and different ways of correcting the confounding factors. The role of chemoradiation has been little investigated in patients with a colostomy for CRC. In the context of several limitations, we considered that the QoL studies would be useful to understand the factors influencing the QoL of patients with colorectal cancer, especially when diagnosed at younger ages.

A comparative study on a sporadic cancer patient group as compared with a hereditary cancer patient group shows that there are no statistically significant differences in QoL, patients from both groups having a high score of QoL [33]. Another study has shown that after segmental resection or extended resection for hereditary colon cancer, the QoL is not significantly different [34].

Knowing the influencing factors of QoL could help identify patients with special needs [35]. The research should use properly validated tools in large-scale studies for better data comparison [5]. The result of such studies might be useful for oncologists, surgeons, psychologists, and pharmacologists, to choose the best therapy protocols to increase QoL in patients diagnosed with CRC at young ages.

The strengths of the present study refer to a group of patients with Lynch syndrome who are young patients, socially active, for whom to maintain a high level of QoL is extremely important even after the oncological therapeutic interventions. The weaknesses of our study refer to the small group, few demographic variables and the non-inclusion in the statistical evaluation of the histopathological and surgical data.

The same team of researchers from our university intends to continue the study on larger groups of patients, in order to better conclude the results and extrapolate the conclusions in the hospital management, population awareness and patients' information, as well as for future projects of other research teams.

\section{Conclusions}

The effects of chemoradiation associated with colostomy in colorectal cancer patients revealed correlations that made the patient to re-analyze the physical and emotional functioning. In our study, impairments of physical and emotional functioning scores were observed. The global score highlighted a moderate decrease in quality of life, both sexes being equally affected.

Supplementary Materials: The following are available online at http://www.mdpi.com/2076-3417/10/18/6585/s1, Table S1: Correlation matrix - items/genders Masculine, Table S2: Correlation matrix—symptoms scale, Table S3: Correlation matrix-emotional functioning scale, Table S4: Correlation matrix-physical functioning scale.

Author Contributions: Conceptualization, V.A.C., R.P., I.M.E., and D.A.; Data curation, M.B., and M.M.; Formal analysis, R.P.; Investigation, M.B., and V.P.; Methodology, V.A.C., M.B., I.M.E., and D.A.; Project administration, V.A.C., and D.A.; Resources, M.M., L.C., and V.P.; Software, M.M.; Supervision, R.P., E.A.C., and D.A.; Validation, V.P.; Visualization, M.B.; Writing—original draft, V.A.C., and L.S.; Writing—review and editing, E.A.C. All authors have read and agreed to the published version of the manuscript.

Funding: This research received no external funding.

Conflicts of Interest: The authors declare no conflict of interest. 


\section{References}

1. Gheorghe, A.-D.; Zob, D.; Stanculeanu, D.-L. Adverse Events of Bevacizumab in Patients with Metastatic Colorectal Cancer. Rev. Chim. 2020, 71, 140-144. [CrossRef]

2. Argillander, T.; Koornstra, J.J.; Van Kouwen, M.; Langers, A.M.; Nagengast, F.M.; Vecht, J.; Cappel, W.H.D.V.T.N.; Dekker, E.; Van Duijvendijk, P.; Vasen, H.F.A. Features of incident colorectal cancer in Lynch syndrome. United Eur. Gastroenterol. J. 2018, 6, 1215-1222. [CrossRef] [PubMed]

3. Chirica, V.; Matei, M.C.; Postolica, R.; Gavrilovici, C.; Azoicai, D. Colorectal Cancer-Epidemiological Perspectives in the Oncogenetic Context. Rev. Med. Chir. Soc. Med. Nat. Iasi. 2015, 119, 821-826.

4. Utescu, C.; Berceanu-Vaduva, D.; Luca, C.T.; Farcas, C.; Dumitrascu, V.; Radulescu, M. Preliminary in vitro Evaluation of a Semisynthetic Oestrogen Polymer-based Formulation. Mater. Plast. 2019, 56, 291-294. [CrossRef]

5. Marventano, S.; Forjaz, M.J.; Grosso, G.; Mistretta, A.; Giorgianni, G.; Platania, A.; Gangi, S.; Basile, F.; Biondi, A. Health related quality of life in colorectal cancer patients: State of the art. BMC Surg. 2013, 13 (Suppl. S2), S15. [CrossRef] [PubMed]

6. Hinganu, D.; Stan, C.I.; Ciupilan, C.; Grigorovici, A.; Bulimar, V.; Cozma, L.C.D.; Hinganu, M.V. Anatomical and Immunohistochemical Evaluation of Colorectal Cancer. Rev. Chim. 2019, 70, 236-238. [CrossRef]

7. Fodor, D.; Suciu, B.A.; Jung, I.; Gurzu, S.; Sipos, T.; Doros, A.; Kobori, L. Transarterial Chemoembolization (Tace) With Lipiodol (R) In Hcc Patientstechnical, Clinical and Imagistic Aspects. Mater. Plast. 2019, 56, 195-198. [CrossRef]

8. Poroch, V.; Lupusoru, C.E.; Lupusoru, R.V.; Gavril, R.S.; Boanca, M. Multidisciplinary Approach to Palliative Care Within the Regional Institute of Oncology Iasi-Physicians' Perception. Rev. Med. Chir. Soc. Med. Nat. Iasi. 2015, 119, 864-872.

9. Poroch, V.; Boanca, M. Palliative Care-Integration Model into Oncological Assistance for the Patients of Regional Institute of Oncology Iasi. Rev. Med. Chir. Soc. Med. Nat. Iasi. 2014, 118, 171-177.

10. Ferlay, J.; Ervik, M.; Lam, F.; Colombet, M.; Mery, L.; Piñeros, M.; Znaor, A.; Soerjomataram, I.; Bray, F. Global Cancer Observatory: Cancer Today; International Agency for Research On Cancer: Lyon, France, 2018; Available online: https://gco.iarc.fr/today/home (accessed on 2 February 2020).

11. Paika, V.; Almyroudi, A.; Tomenson, B.; Creed, F.; Kampletsas, E.O.; Siafaka, V.; Gkika, S.; Mavreas, V.; Pavlidis, N.; Hyphantis, T. Personality Variables Are Associated with Colorectal Cancer Patients' Quality of Life Independent of Psychological Distress and Disease Severity. Psycho Oncol. 2010, 13, 273-282. [CrossRef]

12. Tafreshi, M.Z.; Rassouli, M.; Dabirian, A.; Yaghmaei, F. Quality of life in ostomy patients: A qualitative study. Patient Prefer. Adherence 2010, 13, 1-5. [CrossRef] [PubMed]

13. Gray, N.M.; Hall, S.J.; Browne, S.; MacLeod, U.; Mitchell, E.; Lee, A.J.; Johnston, M.; Wyke, S.; Samuel, L.; Weller, D.; et al. Modifiable and fixed factors predicting quality of life in people with colorectal cancer. Br. J. Cancer 2011, 104, 1697-1703. [CrossRef] [PubMed]

14. Jasperson, K.W.; Tuohy, T.M.; Neklason, D.W.; Burt, R. Hereditary and Familial Colon Cancer. Gastroenterology 2010, 138, 2044-2058. [CrossRef] [PubMed]

15. Dominguez-Valentin, M.; Sampson, J.R.; Seppälä, T.T.; Broeke, S.W.T.; Be, J.-P.P.; Nakken, S.; Engel, C.; Aretz, S.; Jenkins, M.A.; Sunde, L.; et al. Cancer risks by gene, age, and gender in 6350 carriers of pathogenic mismatch repair variants: Findings from the Prospective Lynch Syndrome Database [published correction appears in Genet Med. 2020 Sep;22,1569]. Genet. Med. 2020, 22, 15-25. [CrossRef]

16. Ribic, C.M.; Sargent, D.J.; Moore, M.J.; Thibodeau, S.N.; French, A.J.; Goldberg, R.M.; Hamilton, S.R.; Laurent-Puig, P.; Gryfe, R.; Shepherd, L.E.; et al. Tumor Microsatellite-Instability Status as a Predictor of Benefit from Fluorouracil-Based Adjuvant Chemotherapy for Colon Cancer. N. Engl. J. Med. 2003, 349, 247-257. [CrossRef]

17. Rustgi, A.K. The genetics of hereditary colon cancer. Genes Dev. 2007, 21, 2525-2538. [CrossRef]

18. Seppälä, T.; Pylvänäinen, K.; Evans, D.G.; Järvinen, H.; Renkonen-Sinisalo, L.; Bernstein, I.; Holinski-Feder, E.; Sala, P.; Lindblom, A.; Macrae, F.; et al. Colorectal cancer incidence in path_MLH1 carriers subjected to different follow-up protocols: A Prospective Lynch Syndrome Database report. Hered. Cancer Clin. Pract. 2017, 15, 18. [CrossRef] 
19. Møller, P.; Seppälä, T.T.; Bernstein, I.; Holinski-Feder, E.; Sala, P.; Evans, D.G.; Lindblom, A.; Macrae, F.; Blanco, I.; Sijmons, R.; et al. Incidence of and survival after subsequent cancers in carriers of pathogenic MMR variants with previous cancer: A report from the prospective Lynch syndrome database. Gut 2016, 66, 1657-1664. [CrossRef]

20. Lizdenis, P.; Birutis, J.; Čelkienè, I.; Samalavičius, N.; Kuliavas, J.; Slunskis, V.; Poškus, T.; Jotautas, V.; Poškus, E.; Strupas, K.; et al. Short-Term Results of Quality of Life for Curatively Treated Colorectal Cancer Patients in Lithuania. Medicina 2015, 51, 32-37. [CrossRef]

21. Agheorghiesei, T.D.; Poroch, V. A Possible Theoretical Model of Ethical System Management in Healthcare Institutions, Rethinking Social Action. Core Values 2015. In Proceedings of the 6th Lumen International Conference on Rethinking Social Action Core Values Location, Iasi, Romania, 16-19 April 2015; pp. $33-41$.

22. Toader, E.; Balan, G.G.; Constantinescu, G.; Bulgaru-Iliescu, D. Preventing Malpractice and Medical Litigation in Digestive Interventional Endoscopy by The Use of Empirical Models for The Informed Consent. Rom. J. Leg. Med. 2018, 26, 429-436.

23. Toader, E.; Toader, T. Ethical and Constitutional Values Reflected in Medical Law. Rev. Romana Bioet. 2012, 10, 66-70.

24. Toader, E. Ethics in Medical Technology Education. Rev. Romana Bioet. 2010, 8, 157-162.

25. Agheorghiesei (Corodeanu), D.T.; Poroch, V. Ethical Audit Practice in Hospital Units-The Pillar of the Third Generation of Ethics. Postmod. Open. 2016, 7, 137-147. [CrossRef]

26. Poroch, V.; Agheorghiesei, D.T. A Possible Diagnostic of the State of Health of Ethics Management in the Hospitals in Romania-An Exploratory Study. Postmod. Open. 2018, 9, 225-253. [CrossRef] [PubMed]

27. Van Der Hout, A.; Neijenhuijs, K.I.; Jansen, F.; Van Uden-Kraan, C.F.; Aaronson, N.; Groenvold, M.; Holzner, B.; Terwee, C.B.; Van De Poll-Franse, L.V.; Cuijpers, P.; et al. Measuring health-related quality of life in colorectal cancer patients: Systematic review of measurement properties of the EORTC QLQ-CR29. Support. Care Cancer 2019, 27, 2395-2412. [CrossRef]

28. Reese, J.B.; Finan, P.H.; Haythornthwaite, J.A.; Kadan, M.; Regan, K.R.; Herman, J.M.; Efron, J.; Diaz, L.A., Jr.; Azad, N.S. Gastrointestinal ostomies and sexual outcomes: A comparison of colorectal cancer patients by ostomy status. Support. Care Cancer 2013, 22, 461-468. [CrossRef] [PubMed]

29. Vonk-Klaassen, S.M.; De Vocht, H.M.; Ouden, M.E.M.D.; Eddes, E.H.; Schuurmans, M.J. Ostomy-related problems and their impact on quality of life of colorectal cancer ostomates: A systematic review. Qual. Life Res. 2015, 25, 125-133. [CrossRef] [PubMed]

30. Pereira, M.G.; Figueiredo, A.P.; Fincham, F.D. Anxiety, depression, traumatic stress and quality of life in colorectal cancer after different treatments: A study with Portuguese patients and their partners. Eur. J. Oncol. Nurs. 2012, 16, 227-232. [CrossRef]

31. Altuntas, Y.; Kement, M.; Gezen, C.; Eker, H.; Aydın, H.; Sahin, F.; Okkabaz, N.; Oncel, M. The role of group education on quality of life in patients with a stoma. Eur. J. Cancer Care 2012, 21, 776-781. [CrossRef]

32. Quidde, J.; Pan, Y.; Salm, M.; Hendi, A.; Nilsson, S.; Oechsle, K.; Stein, A.; Nestoriuc, Y. Preventing adverse events of chemotherapy by educating patients about the nocebo effect (RENNO study)—Study protocol of a randomized controlled trial with gastrointestinal cancer patients. BMC Cancer 2018, 18, 916. [CrossRef]

33. Burton-Chase, A.M.; Parker, W.; Donato, K.M.; McCormick, S.; Gritz, E.R.; Amos, C.I.; Lu, K.H.; Lynch, P.M.; Rodríguez-Bigas, M.A.; You, Y.N.; et al. Health-related quality of life in colorectal cancer survivors: Are there differences between sporadic and hereditary patients? J. Patient Rep. Outcomes 2018, 2, 21. [CrossRef] [PubMed]

34. Pollett, W.G.; Marion, K.; Moeslein, G.; Schneider, C.; Parry, S.; Veysey, K.; Bissett, I.; Jones, I.; Macrae, F. Quality of life after surgery in individuals with familial colorectal cancer: Does extended surgery have an adverse impact? ANZ J. Surg. 2013, 84, 359-364. [CrossRef] [PubMed]

35. Lunguleac, T.; Azoicai, D.; Manole, A.; Patrascu, A.; Moscalu, M. The Contribution of Stress Level in Modifying the Cardiometabolic Risk in a Population Cohort from North-East Romania. Rev. Chim. 2019, 70, 1071-1075. [CrossRef]

(C) 2020 by the authors. Licensee MDPI, Basel, Switzerland. This article is an open access article distributed under the terms and conditions of the Creative Commons Attribution (CC BY) license (http://creativecommons.org/licenses/by/4.0/). 